\title{
DISEÑO E IMPLEMENTACIÓN DE HMI Y SCADA APLICADOS A LA EMULACIÓN DIDÁCTICA DE UN PROCESO DE ENVASADO
}

\author{
(Design and implementation of HMI and SCADA \\ applied to a didactic emulation of a packing process)
}

\section{Manuel Alejandro Bohórquez Dallos, Carlos Noé Salamanca Amaya}

Universidad Pedagógica y Tecnológica de Colombia. Grupo de Investigación DSP

manuelalejandro10@gmail.com, carlos.salamanca505@hotmail.com.

(Recibido19 de Noviembre de 2012 y aceptado 13 de Marzo de 2013)

\section{Resumen:}

Este documento muestra el diseño e implementación de una interfaz humano maquina (HMI) en una pantalla táctil industrial programada en el software Galileo $\AA$, y un sistema SCADA (Supervisory Control and Data Acquisition) en computador, que usa el software CoDeSys ${ }^{\circledR}$, con los que se realizan supervisión, control y adquisición de datos correspondientes a sensores analógicos y digitales, aplicados a la emulación de un proceso de envasado. La supervisión y el control de las señales se hacen con el protocolo CANopen. El sistema desarrollado permite al usuario interactuar directamente con la emulación del proceso, accionando elementos de este en modo remoto en el computador, y en modo local con la pantalla táctil. El SCADA y la HMI están embebidos en una función en CoDeSys ${ }^{\circledR}$, que el usuario puede usar como herramienta de aprendizaje para efectuar la automatización de la emulación del proceso.

Palabras clave: CANopen, emulación didáctica, HMI, pantalla táctil, PLC, proceso de envasado, SCADA, sensores industriales.

\section{Abstract:}

This paper shows the design and implementation of a Human Machine Interface (HMI) in an industrial touch screen programmed in the Galileo ${ }^{\circledR}$ software and SCADA (Supervisory Control and Data Acquisition) computer system using software CoDeSys ${ }^{\circledR}$, with those supervision, control and data acquisition for analog and digital sensors are made, applied to the emulation of a packaging process. The supervision and control of the signals are performed with the CANopen protocol. The developed system allows the user to interact directly with the emulation of the process, triggering elements of it in the computer remotely and locally using the touch screen. The SCADA and HMI are embedded in a CoDeSys ${ }^{\circledR}$ function that the user can use as a learning tool for automating the process emulation.

Keywords: CANopen, didactic emulation, HMI, industrial sensors, packing Process, PLC, SCADA, touch screen.

\section{INTRODUCCIÓN}

Si bien existen muchas formas de llevar a cabo la supervisión y control de procesos, ya sea en computadores o en elementos dedicados exclusivamente a esta labor (como es el caso de las pantallas táctiles), para la supervisión y control local de procesos se destacan las pantallas táctiles industriales debido a su funcionalidad y al uso espacial reducido. Adamo et al. (2005) destacan que hasta hace poco tiempo los equipos que permitían la interacción de un operador humano con una maquina simple o con una planta completa eran construidos por medio de componentes eléctricos y electromecánicos en forma de paneles sinópticos, a menudo muy complicados y engorrosos, con pulsadores, lámparas, interruptores, etc.; aunque desde hace pocos años esos paneles han sido sustituidos por enormes interfaces virtuales sofisticadas mostradas en CRT o monitores LCD en forma de paneles interactivos gráficos. Esas instalaciones son conocidas como sistemas HMI (Human Machine Interface). Montalvo y Morocho (2011) indican que el uso de módulos didácticos tiene como objetivo principal ofrecer al estudiante la posibilidad de verificar en la práctica todo lo que se explica en clase, de tal forma que con el trabajo que plantea se puedan desarrollar prácticas de control y automatización de procesos industriales de forma sencilla, rápida y segura. De acuerdo con este contexto y con la disposición de equipos en el laboratorio de automatización industrial de la UPTC Sogamoso, se realiza un sistema SCADA aplicado a la emulación de un proceso de envasado en una pantalla táctil industrial y en un sistema de visualización en computador, que permite al usuario interactuar con las caracteristicas reales de un proceso al hacer la automatización de este, sin necesidad de tener la implementación física, pero con instrumentación, equipos de visualización, control y adquisición de señales que se usarían en el proceso real. Inicialmente se establecen las variables del proceso y se miden con sensores industriales (analógicos y digitales). Dichas señales se adquieren desde un módulo de expansión de entradas salidas XNE-GWBR-CANOPEN (donde se conectan todos los sensores), por medio del protocolo CANopen. 
El módulo de expansión de entradas, por su parte, se comunica con un módulo central, desde donde se controla la emulación del proceso. Este módulo está constituido por un PLC y una pantalla táctil industrial, y se conecta al computador. Finalmente, como elemento actuador de la emulación del proceso se usan cuatro motores conectados a un módulo de arranque directo, el cual tiene conexión por conectores RJ45, lo cual permite su conexión con los demás módulos, de acuerdo con la topología de red adecuada para el protocolo CANopen.

Con el programa implementado en el PLC, el usuario realiza el control de la emulación del proceso, usando una función desarrollada en CoDeSys ${ }^{\circledR}$ por los autores, de la cual se leen los parámetros de salida, y, dependiendo de las lecturas de estos, se ejercen acciones de control en los parámetros de entrada de la función, obteniendo de esta forma cambios en el funcionamiento de la emulación del proceso. De este modo, el usuario dispone de una herramienta de aprendizaje para efectuar la automatización de un proceso, programando el PLC sin necesidad de hacer todo el sistema SCADA ni la configuración de módulos o la comunicación de estos (ya se hace internamente en la función). El módulo de PLC-pantalla táctil tiene la posibilidad de conectarse por puerto Ethernet con un computador en el que está implementado un sistema SCADA en el software CoDeSys ${ }^{\circledR}$, igualmente hay una conexión entre el PLC y la pantalla táctil. En este último elemento se implementa otro sistema en el software Galileo ${ }^{\circledR}$.

Con el desarrollo del proyecto se busca principalmente entregar la emulación del proceso, pero se establece un aporte adicional, que es enseñar al usuario, paso a paso, como se lleva a cabo la emulación de procesos sencillos con los equipos descritos anteriormente. Para esto se establecen cuatro procesos introductorios correspondientes a cuatro emulaciones sencillas que aplican los conceptos básicos que se aplicarían en la realización de una emulación de proceso grande.

\section{PROCESOS INTRODUCTORIOS}

Aunque se pudo ejecutar directamente la función para la emulación del proceso, se decidió inicialmente hacer unas guías instructivas para el desarrollo de sistemas de supervisión en pantalla táctil con la emulación de procesos sencillos, con los que se busca introducir progresivamente al usuario en el manejo de todos los elementos usados en la emulación de un proceso grande, pero que ofrecen mayor sencillez en la explicación de los mismos. Esto se hace teniendo en cuenta a Bejan et al. (2009), quienes muestran un grupo de laboratorios prácticos para introducir gradualmente los elementos principales de un SCADA, y, por último, desarrollan una aplicación para controlar un motor de inducción en modo manual y automático con una pantalla táctil.

Antes de describir los procesos introductorios, hay que tener en cuenta algunas caracteristicas básicas de los sistemas SCADA, en los que se basan las emulaciones desarrolladas. Los sistemas SCADA aplicados a cualquier proceso, apoyan su funcionamiento en el envío y recepción de señales analógicas y digitales, pero hay aspectos adicionales que deben tenerse en cuenta.
Rodríguez (2007) indica las siguientes prestaciones que debe tener un sistema SCADA: monitorización, supervisión, adquisición de datos en los procesos de observación, visualización de los estados de las señales del sistema (alarmas y eventos), el mando, grabación de acciones o recetas, garantía de seguridad de datos, garantía de seguridad en los accesos. Se resalta que las anteriores prestaciones son aplicables tanto a sistemas SCADA en pantallas táctiles, como a sistemas en computador.

Los procesos introductorios se realizan tanto en la pantalla táctil, usando el software Galileo ${ }^{\circledR}$, como en el computador, usando el software CoDeSys ${ }^{\circledR}$. Con la emulación de los procesos introductorios, se aprovechan los recursos disponibles en un sistema de supervisión en pantalla táctil, como desarrollos de menús, supervisión y control de señales analógicas y digitales (User Manual Galileo 4.2 Software, 2012), que no han sido explorados profundamente en los equipos de la UPTC Sogamoso. A continuación se describen las caracteristicas fundamentales de los procesos introductorios, $\mathrm{y}$, posteriormente, se explican la estructura y funcionamiento de la función desarrollada por los autores para la emulación del proceso de envasado.

\subsection{Proceso menús arranque directo}

La Figura 1 corresponde a la emulación del arranque directo de tres motores, usando un módulo de arranque directo, un módulo de pantalla táctil y PLC comunicados por protocolo CANopen. El estudiante, teniendo en cuenta lo explicado por Bohórquez y Salamanca (Guía 1, 2012) realiza paso a paso la emulación del arranque directo con los elementos indicados al comienzo.

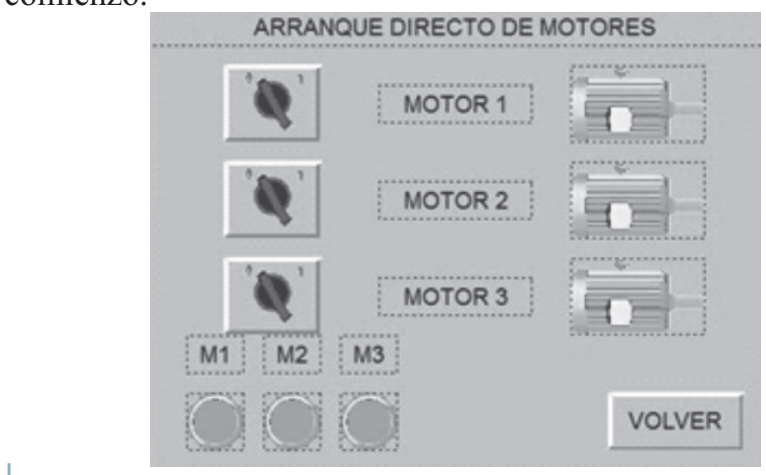

Figura 1. Interfaz en pantalla táctil para emulación de proceso de arranque directo de motores.

\subsection{Proceso de corte de madera}

La Figura 2 corresponde a la emulación de un proceso de corte de madera, usando un módulo de arranque directo (correspondiente al motor de la sierra), dos sensores industriales de proximidad (uno capacitivo para madera y uno inductivo para detectar la sierra), un módulo de expansión de entradas-salidas XNE-GWBR-CANOPEN (para adquirir señales digitales), $\mathrm{y}$, adicionalmente, un módulo de pantalla táctil y PLC comunicados por protocolo CANopen. El estudiante realiza paso a paso (Bohórquez, Salamanca, Guía 2, 2012) la emulación del proceso de corte de madera con los módulos y sensores indicados anteriormente.
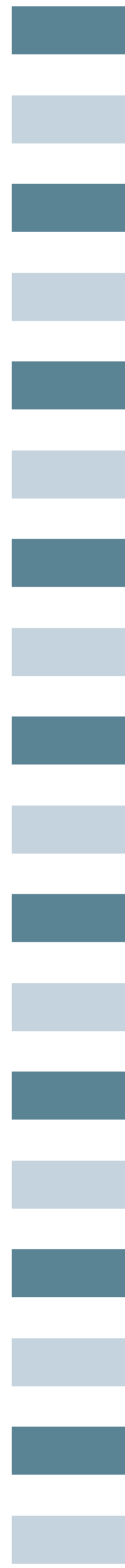


\subsection{Proceso de enrollado-desenrollado de dos carretes}

Es un proceso de enrollado de un material en un carrete, al cual está conectado un motor (este motor se emula con uno de los motores del módulo de arranque directo). El material se recoge de otro carrete, en el que se está midiendo continuamente el grosor del material que se va desenrollando. En este caso se usa un sensor analógico de ultrasonido de distancia. El sistema correspondiente al proceso implementado en pantalla táctil, se muestra en la Figura 3. Bohórquez y Salamanca (Guía 3, 2012) le indican paso a paso al estudiante como hacer la emulación del proceso en pantalla táctil.

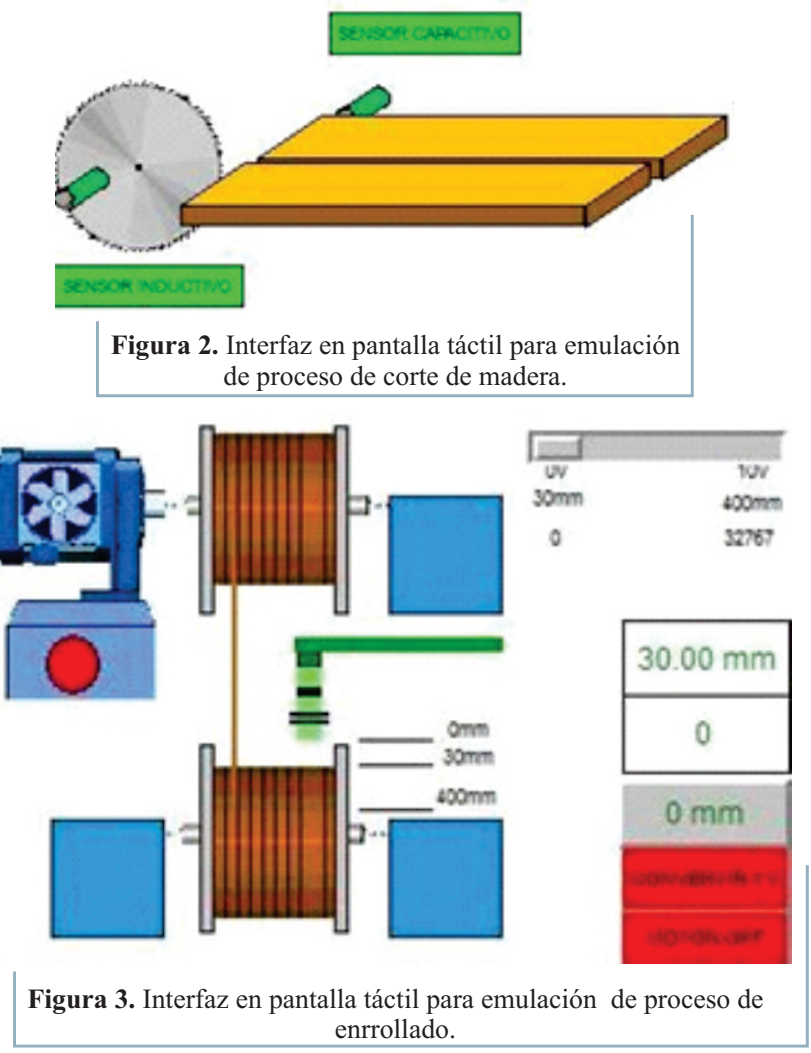

2.4 Proceso conjunto tanques-sistema posicionamiento lineal

El sistema, para este caso, consta de un menú de dos opciones realizadas en pantalla táctil, cada una de estas opciones corresponde a una emulación. La emulación uno pertenece a un sistema en el que se supervisa la posición de un elemento que corresponde a un sensor de posición lineal inductivo PMI360F110-IU-V1 (Figura 4). Este sensor tiene un elemento metálico que, a medida que se mueve, ocasiona cambios en una señal analógica (voltaje), y se halla conectado a una de las entradas analógicas del módulo de expansión de señales XNE-GWBRCANopen. Adicional al sistema explicado anteriormente, se adjunta en la misma aplicación (tanto en la pantalla táctil como en CoDeSys ${ }^{\circledR}$ ) un sistema de supervisión de nivel para un tanque que contiene líquido (ver Figura 5). El principio de funcionamiento es el mismo, pero es un sensor que, en este caso, es de tipo de ultrasónico de distancia UB800-18GM40AU-V1, mide el nivel del tanque, (Bohórquez \& Salamanca, Guía 4, 2012).

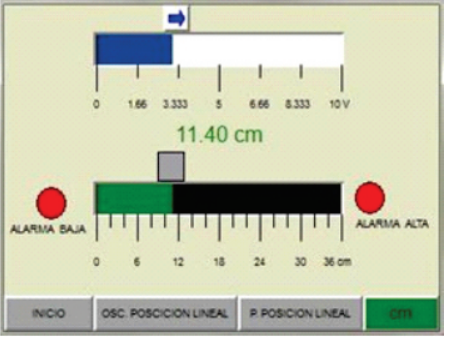

Figura 4. Interfaz en pantalla táctil para visualización de posición lineal

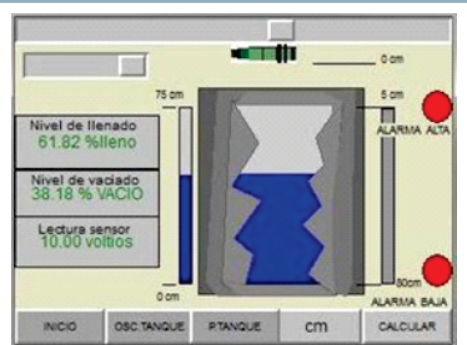

Figura 5. Interfaz en pantalla táctil para emulación del proceso llenado tanque.

\section{EMULACIÓN PROCESO ENVASADO}

Goodwin et al. (2011) destacan que el término emulación se utiliza en lugar de simulación, para reflejar la inclusión de efectos del mundo real no ideales, con lo cual, los resultados obtenidos en este tipo de procesos no siempre coinciden con las predicciones puramente teóricas que ofrece el diseño, pero es más acorde con la realidad del diseño de ingeniería. La emulación brinda la ventaja de incluir efectos reales no considerados en la teoría, no requiere de la implementación total de los procesos, representando un ahorro económico y de tiempo, además familiariza al estudiante con el funcionamiento de procesos reales, con los que se enfrentará en su área de desempeño profesional en la automatización.

\subsubsection{Función}

El objetivo fundamental del proyecto es entregar una aplicación correspondiente a la emulación de un proceso, a fin de que el usuario tenga una herramienta para automatizar, además sirve como elemento de fortalecimiento de conceptos para la programación de PLC, contextualizando al estudiante en el manejo de un sistema que tiene un uso industrial. El manejo de esta aplicación se da desde la pantalla táctil o desde el computador.

De acuerdo con el software CoDeSys ${ }^{\circledR}$ y con los módulos disponibles, se encontró que el modo más adecuado y que se adaptaba a las características de los elementos que se tienen, es el uso de una función. CoDeSys ${ }^{\circledR}$ tiene un gran número de herramientas para diversas aplicaciones con PLC, pero la mayoría de ellas se implementa directamente usando funciones, en las que se dispone de unas entradas y unas salidas que se usan de acuerdo con la necesidad del usuario y de la aplicación (User Manual for PLC Programming with CoDeSys $2.3,2012)$. Un ejemplo claro de estas funciones es la función que se usa para el manejo de la HMI (pantalla táctil), la cual controla la comunicación entre el PLC y la HMI (Automatización BP, 2012) 
3.1.2 Descripción. La función desarrollada por los autores, es similar estructuralmente a las funciones internas de CoDeSys ${ }^{\circledR}$, pero gobierna todas las características de la emulación del proceso y está implementada en texto estructurado (ST). Este es un lenguaje estructurado de alto nivel, similar a Pascal y C, corresponde a uno de los dos lenguajes basados en texto, definidos por el estándar IEC 11313 (Parr, 2003).

La función consta de 44 parámetros en total. De esos, 33 corresponden a entradas y 11, a salidas. Estos parámetros están asignados tanto a un sistema SCADA en CoDeSys ${ }^{\circledR}$, como a uno en la pantalla táctil y a los elementos de los módulos usados (módulo de expansión de entradas y módulo de arranque directo). Los parámetros de entrada accionan alguna componente de la emulación del proceso (por ej., motores, válvulas) o asignan valores numéricos que se pueden visualizar en las pantallas SCADA que tiene el proceso (por ej., número de envase por llenar). Para el caso de los parámetros de salida de la función, en estos se guardan los valores que se insertan desde la visualización de CoDeSys ${ }^{\circledR}$ o desde el SCADA usado en la HMI, tales como accionamientos en botones, inserción de parámetros del proceso, entre otros.

Los sistemas de alarma son básicos para cualquier proceso y deben indicarle al operario claramente el problema en desarrollo a tiempo, a fin de que este tome la decisión indicada para resolver el problema. Un buen sistema de alarma debe cumplir ciertos requisitos que se resaltan a continuación: las alarmas deben ser claras, únicas, concisas y no ambiguas; deben tener definida una respuesta del operador; deben ser implícitamente confiables; deben decirle a los operadores lo que ellos necesitan conocer (información relevante del funcionamiento del proceso); y deben activarse cuando el operador tenga tiempo de responder (Parr, 2003).

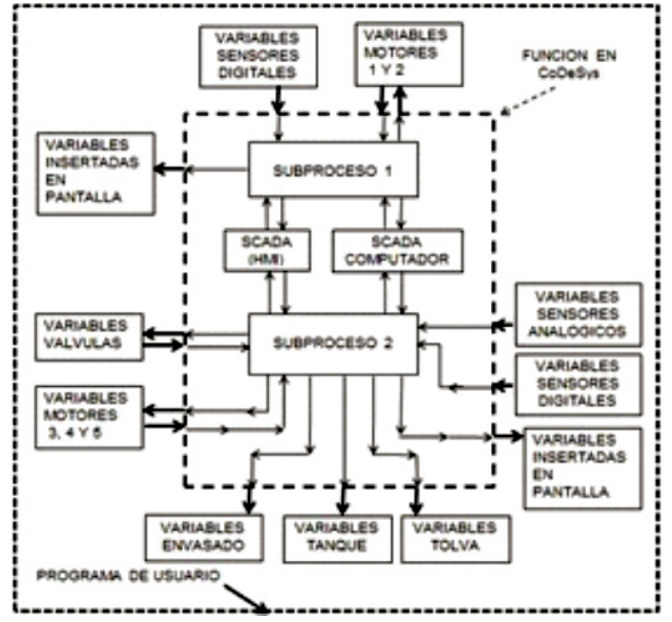

Figura 6. Esquema interno de función usada para emulación de proceso de envasado y parámetros disponibles para desarrollo del programa de usuario.

Estos puntos clave en cuanto a las alarmas, se aplican en la emulación del proceso, donde se busca mostrar al usuario los indicadores de alarma o error en el manejo del proceso. Para esto, la función desarrollada internamente y de acuerdo con las características reales de funcionamiento del proceso, determina 15 indicadores de error (banderas de error), que se activan automáticamente en caso de que la operación del proceso sea incorrecta (Bohórquez \& Salamanca, Guía 5).
El propósito de esto es mostrarle al usuario qué efectos no deseados se observarían en el proceso en la realidad, entregándole un elemento de guía para evitar y corregir estos errores de operación. En otras palabras, la idea es que con el programa que el usuario desarrolle, ninguno de esos indicadores de error se active.

Internamente, en la función se hace la configuración del llamado de la pantalla táctil, se realiza el respectivo envío de los TAG necesarios para la comunicación bidireccional con la HMI (usados en la interfaz de la pantalla táctil), y se asignan los TAG al sistema SCADA en CoDeSys ${ }^{\circledR}$. La función desarrollada considera las características físicas que tendría el proceso en la realidad, tales como movimientos de motores, movimientos de bandas transportadoras, movimientos de material, líquidos, y circulación de líquidos y fluidos, elementos que hacen más real e interactiva la emulación del proceso.

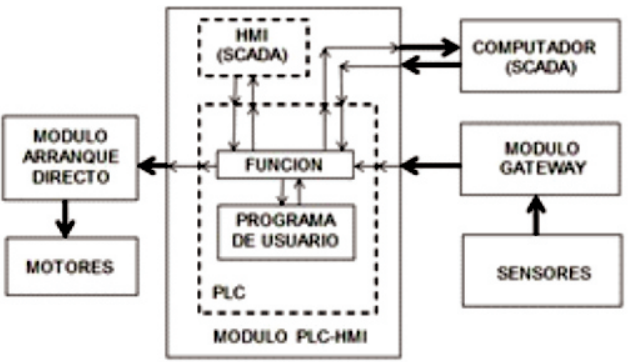

Figura 7. Esquema de la emulación del proceso y uso de la función desarrollada.

3.1.3 Uso de la función. Para usar la función en CoDeSys ${ }^{\circledR}$, primero se seleccionan las entradas del módulo de expansión de entradas (previa configuración de la red en CANopen con los elementos que la componen), después se llama la función y, posteriormente, ya se puede desarrollar el programa para controlar toda la emulación del proceso usando texto estructurado (ST). La Figura 6 indica el esquema interno de la función y el programa de usuario con todos los parámetros de entrada-salida que se disponen para automatizar la emulación del proceso. Teniendo en cuenta esto, el usuario solamente conecta sensores en las entradas adecuadas del módulo de expansión, hace la conexión al puerto Ethernet, tanto del módulo de expansión de entradas (donde están conectados los sensores) como del módulo de arranque directo (motores que emulan los actuadores del proceso), de acuerdo con la topología de red correspondiente que se requiere para una conexión por CANopen. Con esto, el usuario ya puede poner en funcionamiento el proceso, de acuerdo con el esquema indicado (Figura 7).

La función desarrollada tiene la ventaja que puede ser usada con los elementos conectados (módulos, sensores) o sin los elementos conectados, lo que le ofrece al usuario un proceso que puede usar tanto para simulación (elementos no conectados) como para emulación (elementos conectados).

La función implementada está conectada a un sistema SCADA, tanto en el programa de CoDeSys ${ }^{\circledR}$, como en la pantalla táctil, los dos lados cumplen la misma función y se observan iguales características. El sistema de visualización de CoDeSys ${ }^{\circledR}$ ofrece una herramienta de seguridad para el manejo del proceso mediante contraseñas, donde hay una distribución de permisos para cada parte del programa.
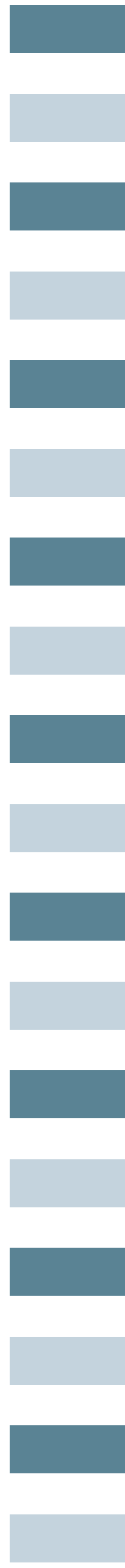
En este caso se dejan permisos para lectura de la función, pero no para la modificación, así que el usuario tiene la posibilidad de leer y accionar los elementos que hay en el SCADA, pero no de modificarlos. De la misma forma, en la HMI (pantalla táctil), el software Galileo ${ }^{\circledR}$ con el que se desarrolla la interfaz, se implementa en una pantalla inicial que muestra varias cuentas de usuario, que, dependiendo de la que se seleccione, posee permisos de acceso para ciertas partes del proceso.

\subsection{Proceso}

La función ejecutada en CoDeSys ${ }^{\circledR}$, describe el proceso de envasado de sustancias sólidas y líquidas. El sistema de supervisión, control y adquisición de datos, se efectúa para este proceso en una pantalla táctil y de la misma forma en el sistema de visualización que dispone CoDeSys ${ }^{\circledR}$. De este modo, el usuario tiene la posibilidad de accionar e insertar elementos del proceso, tanto desde la pantalla táctil (modo local) como desde la interfaz en CoDeSys ${ }^{\circledR}$ (modo remoto). En los dos casos, el usuario puede observar características de funcionamiento del proceso en la realidad, como movimiento de bandas transportadoras, niveles, etc.

En el proceso hay dos tipos de envase, el primero tiene una capacidad de almacenamiento V (envase grande), el segundo tiene una capacidad de almacenamiento $V / 2$ (envase pequeño). Para el caso de los envases grandes (capacidad V), se debe depositar sustancia sólida y líquida a la vez. Para el evento de los envases pequeños, solamente se debe envasar sustancia líquida. De la misma forma en que se implementó la supervisión del proceso en el sistema de visualización en CoDeSys ${ }^{\circledR}$, se efectúa en la pantalla táctil, para todos los subprocesos que se indican a continuación.

El proceso de envasado se divide en varios subprocesos. En seguida se resaltan las características y los requerimientos para cada uno de ellos.

3.2.1 Clasificación de envase. El proceso de envasado está alimentado por dos bandas transportadoras. Cada una de ellas tiene su respectivo motor que se controla por arranque directo remoto, mediante red CANopen (correspondiente al módulo de arranque directo del laboratorio de la UPTC Sogamoso). Una de las bandas entrega envase grande (banda 1) y la otra, envase pequeño (banda 2). La banda 2 trae envase pequeño de un proceso anterior $\mathrm{y}$, a la vez, es la banda trasportadora que suministra el envase para el envasado. La banda 2 está ubicada perpendicularmente a la banda 1 (ver Figura 8), de tal modo que puede existir un choque de envase (la función desarrollada en CoDeSys ${ }^{\circledR}$ tiene un indicador para estos choques). Se asume que con el impulso de la banda 1, basta para que el envase pequeño (proveniente de la banda 1) quede en la banda 2. El objeto fundamental del control de esta parte del proceso, es evitar que choquen los envases en el cruce de las bandas. Para esto se dispone de tres sensores de proximidad en el cruce de las dos bandas, de tal modo que se pueda conocer la presencia de envase en las dos bandas, de acuerdo con estas lecturas se determina si se mueve la banda 1 (motor 1 del módulo de arranque directo) o la banda 2 (motor 2 del módulo de arranque directo).
Para el proceso hay un número limitado de envases por llenar, este límite se inserta en la HMI (pantalla táctil) y en el sistema de visualización de CoDeSys ${ }^{\circledR}$ (SCADA en el computador), de manera que cuando se cumpla este límite, se debe detener todo el proceso. La detección del envase y del tamaño de este, se hace con dos sensores de proximidad, de tal forma que en el cruce de la banda 2 con la banda 1 , se puede conocer si hay un envase grande o un envase pequeño. La idea es que con el control se envíe un envase grande y después un envase pequeño, hasta completar el número de envases grandes y de envases pequeños que se insertó.

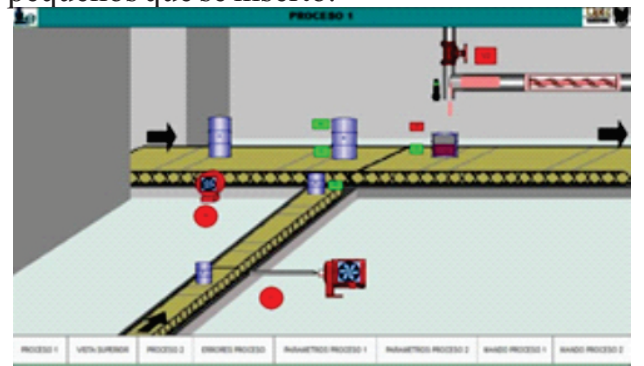

Figura 8. Ubicación de las bandas transportadoras para distribución de envase en CoDeSys ${ }^{\circledR}$.

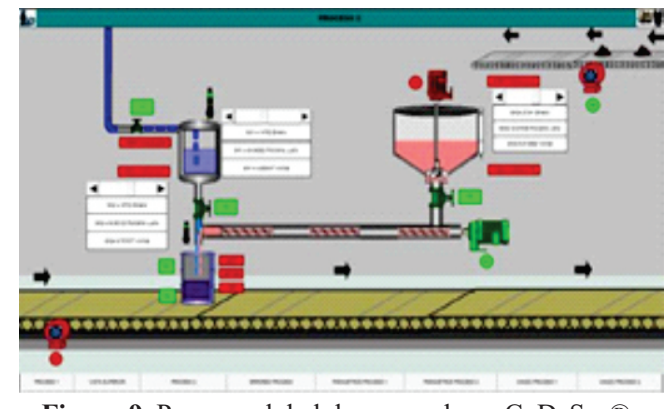

Figura 9. Proceso global de envasado en CoDeSys ${ }^{\circledR}$.

3.2.2 Proceso de envasado. La emulación del proceso de envasado de material consta de un tanque y de una tolva (Figura 9). El tanque contiene el líquido que se va a aplicar, y la tolva, el material para ser molido que se quiere aplicar. El esquema de la Figura 9 también se implementa en la pantalla táctil.

3.2.3 Llenado del tanque de líquido. Este tanque contiene el líquido que se desea depositar en los envases. A este subproceso se le aplica líquido desde una fuente externa que siempre le entrega un flujo constante, la entrada del líquido al tanque se controla con la apertura de la válvula V1 (Figura 10). El sistema le indica al operario si el tanque está lleno y aún le está aplicando líquido, o si está vacío y no se está aplicando. Las válvulas se pueden abrir o cerrar manualmente haciendo clic sobre ellas (en los sistemas SCADA) o en una pantalla desde la que se puede efectuar un control manual del proceso mediante botones correspondientes a las válvulas y motores del proceso.

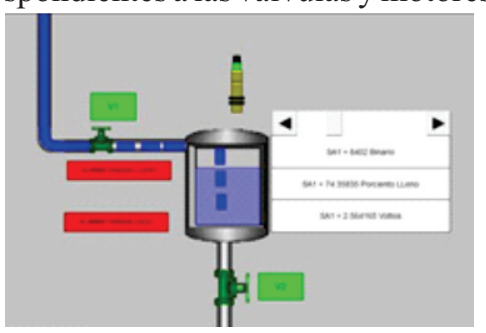

Figura 10. Llenado de tanque de líquido en CoDeSys ${ }^{\circledR}$. 
El objetivo de este subproceso es que el programa en el PLC que haga el usuario para automatizar la emulación, mantenga el nivel del tanque en un intervalo sin que llegue a desocuparse o llenarse totalmente, ya que esto afectaría el llenado del envase, que es el proceso que depende de este subproceso. Para esto se muestran dos indicadores ALARMA TANQUE LLENO y ALARMA TANQUE VACÍO (ver Figura 10), que el usuario activa mediante programa en el PLC, para saber en qué momento el nivel sube o baja de un valor deseado. Estos corresponden a dos valores que el usuario inserta en el sistema (por teclado desde el computador en CoDeSys ${ }^{\circledR}$ o desde la pantalla táctil). Estos valores son porcentajes del volumen del tanque, (por ej., el indicador ALARMA TANQUE LLENO se enciende, si el nivel del tanque llega a ser mayor o igual que el valor insertado como límite alto del tanque, y el indicador ALARMA TANQUE-VACÍO se enciende, si el nivel del tanque es menor que el valor insertado como límite bajo del tanque).

La medición del nivel de este tanque se emula con un sensor ultrasónico UB800-18GM40A-U-V1 (rango de medición de $50 \mathrm{~mm}$ a $800 \mathrm{~mm}$, con lo cual el tanque tiene una altura de 75 $\mathrm{cm}$ ) ubicado en la parte superior del tanque. La pantalla, tanto en la visualización de CoDeSys ${ }^{\circledR}$ como la desarrollada para la pantalla táctil, indica tres lecturas de las que se puede disponer al llamar la función en CoDeSys ${ }^{\circledR}$, que son la lectura del sensor en binario (la lectura que entrega al convertir la señal analógica de 0 a $10 \mathrm{~V}$, proveniente del sensor en una señal digital de 0 a 32767 en binario), la otra lectura es el porcentaje de llenado del tanque (es una medida general que va indicando el aumento o disminución del volumen del tanque) y la última lectura es el voltaje que está entregando como salida el sensor (es un valor de 0 a $10 \mathrm{~V}$ ). Estas tres lecturas se calculan internamente en la función desarrollada en CoDeSys ${ }^{\circledR}$ usando solamente la lectura binaria que se obtiene del sensor.

3.2.4 Llenado de tolva. El segundo subproceso del que se alimenta la emulación del proceso de llenado, es la tolva. A este lugar llega material sólido que se deposita mediante la banda manejada por el motor M5 (ver Figura 11), material que debe ser molido en la tolva (no se puede aplicar material sólido en el sinfín, ya que lo atascaría). La tolva debe ir llenándose al aplicar material sólido con la banda del motor M5, de tal modo que el nivel de llenado de la tolva con sólidos cambia. La medición del nivel de la tolva se emula con un sensor de posición inductivo PMI360-F110-IU-V1 (rango de detección de 0 a $360 \mathrm{~mm}$, salida de 0 a $10 \mathrm{~V}$ ).

Debe tenerse presente que cuando el nivel de llenado con sólido de la tolva esté en un nivel seguro (ni lleno ni vacío), hay que abrir la válvula V3 y encender el motor M4 (motor de molido, ver Figura 11), que permitirá moler el sólido y depositarlo en el tornillo sinfín que está conectado al motor M3.

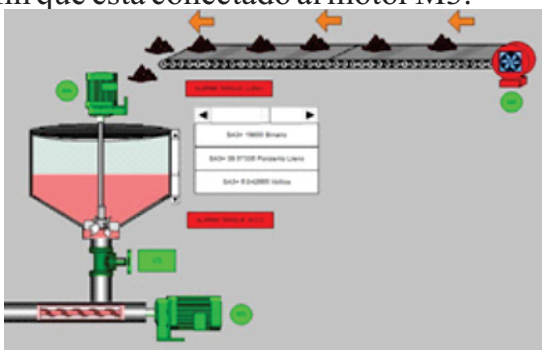

Figura 11.Subproceso llenado de tolva en CoDeSys $\Re$.
Es importante medir el nivel de llenado de la tolva, ya que indica el momento en que hay que aplicar material sólido para ser molido (cuando la tolva esté próxima a desocuparse), o en qué momento hay que dejar de aplicar material sólido (cuando la tolva esté próxima a llenarse). De la misma forma que en el subproceso del tanque, se entregan tres lecturas de la señal del sensor que indican la lectura del sensor en binario, el porcentaje de llenado de la tolva y el voltaje que entregaría el sensor; también se incluyen dos indicadores de nivel del tanque, que se encienden si el nivel sube de un punto determinado (insertado por el usuario), que para la tolva es ALARMA TANQUE LLENO, y para el caso en que el nivel de la tolva baje de cierto valor (también insertado por el usuario), se llama ALARMA TANQUE VACÍO.

3.2.5 Llenado de envase. Como resultado de los dos subprocesos anteriores, se llenan los envases teniendo en cuenta que los envases grandes se llenan con sustancia sólida y líquida a la vez, y los pequeños, con sustancia sólida solamente. La detección del tipo de envase se hace ubicando dos sensores de proximidad en el lugar exacto donde se hace el envasado (ver Figura 12). Estos sensores se conectan a las entradas digitales del módulo de expansión de entradas. La detección del envase se emula con los sensores de proximidad capacitivos CCN15-F64-E2 y CBN5-F46-E2. Para la emulación de la medición del nivel de llenado del envase, se ubica un sensor ultrasónico UB400-12GM-U-V1 que tiene un rango de 30 a $400 \mathrm{~mm}$, con este sensor se emula la medición del nivel de llenado de los dos tipos de envase (grande y pequeño). De la misma forma que los sensores de proximidad, el sensor ultrasónico se conecta a los puertos de entrada, pero analógicos del módulo de expansión de entradas. La lectura del sensor se entrega como en los anteriores subprocesos, en binario, en porcentaje de llenado del envase y en voltaje que entregaría el sensor. Un parámetro de este subproceso es el nivel de llenado (porcentaje de llenado) para el envase (hay un nivel de llenado para el envase grande y otro para el envase pequeño). Es un parámetro que se puede escribir ya sea en la HMI o en la pantalla de visualización de CoDeSys ${ }^{\circledR}$.

Hay tres indicadores (banderas de tipo digital) de nivel, vacío, llenando y lleno, que los puede encender el usuario dependiendo del nivel en que se encuentre el envase (ver Figura 12).

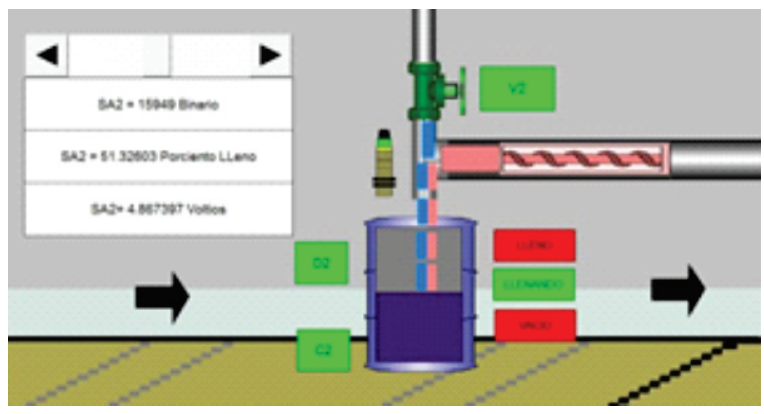

Figura 12. Subproceso llenado de envase CoDeSys ${ }^{\circledR}$.

Como condiciones para este subproceso, no se debe seguir depositando material molido ni líquido, en el caso en que ya esté lleno el envase. No se debe encender el motor M2, en el caso que no se haya llenado el envase en el valor deseado.
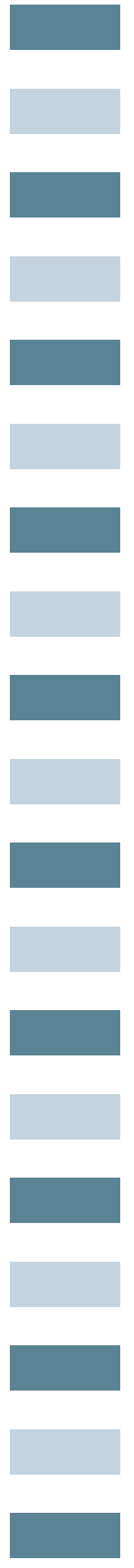
El depósito de elemento líquido se hace activando la válvula V2, el material sólido solo puede aplicarse si el sinfín está girando, para lo cual debe estar encendido M3 (garantizando que haya un nivel adecuado en la tolva, con M4 moviéndose, y V3 abierta).

3.2.6 Parámetros de emulación del proceso. La emulación del proceso le brinda al usuario la posibilidad de tener un control manual y un control automático del mismo. Para el caso del control automático, el usuario usa las señales de sensores en un programa que debe ejecutar él mismo para control de la emulación del proceso (con previo llamado de la función desarrollada en CoDeSys ${ }^{\circledR}$ para la emulación del proceso), con el cual activa los actuadores de la emulación y mantiene las variables de proceso dentro de los rangos adecuados. Los efectos del control que realiza el usuario se observan en el sistema SCADA (tanto en la pantalla táctil como en el computador). Para el caso del control manual, el usuario dispone en el sistema SCADA (tanto en la pantalla táctil como en el computador) de pantallas en las que puede accionar manualmente mediante botones los actuadores de la emulación del proceso. Los efectos sobre el proceso son los mismos si se hiciera el control de forma automática, solo que esta vez el usuario observa las lecturas de los sensores y de acuerdo con esto acciona manualmente los actuadores del proceso. La emulación del proceso tiene dos pantallas para el control manual, correspondientes a cada uno de los subprocesos del sistema de envasado (clasificación de envase, y envasado de material). La pantalla para el subproceso 1, se muestra en la Figura 13, posee botón de start, stop, accionamiento de motores y un selector para determinación de modo manual y de modo automático (el usuario usa estos botones en el programa principal teniendo en cuenta que a cada uno de ellos le corresponde una variable en la función desarrollada). Cada una de las pantallas de mando manual posee un botón de start y uno de stop, que el usuario usa en el programa que implementa, para iniciar el proceso y para detenerlo, en caso de parada de emergencia. Las pantallas de mando manual también se presentan en pantalla táctil, de modo que el usuario tiene la posibilidad de accionar los actuadores y botones del sistema también desde la pantalla táctil (entregando un control local del proceso).

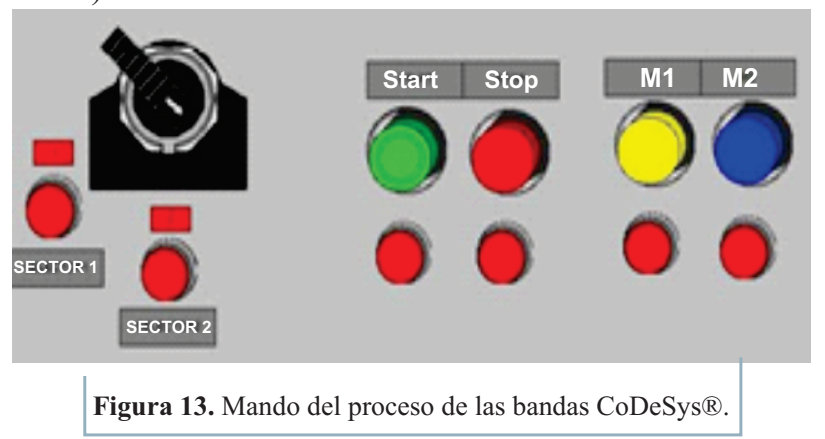

Otro elemento disponible para el usuario, es la inserción de parámetros necesarios para la emulación del proceso, tales como el número de envases por llenar, porcentajes de llenado, límites en el tanque y tolva, entre otros. Para estos parámetros, el usuario dispone de pantallas desde las que puede insertarlos, tanto en la pantalla táctil como en el sistema de visualización de CoDeSys ${ }^{\circledR}$.

\section{PROTOCOLO DE COMUNICACIÓN PARA LOS MÓDULOS}

Los módulos usados para la implementación de la emulación del proceso y el desarrollo de los procesos introductorios, se comunican usando el protocolo CANopen. Los módulos de PLC-pantalla táctil y módulo de expansión de entradas-salidas poseen cada uno dos conectores RJ45, uno corresponde a una entrada y el otro a una salida; aunque se aclara que el módulo de arranque directo posee solamente un puerto de entrada, ya que es el módulo que internamente tiene un interruptor que se puede ajustar para terminar la red. La topología de red usada para la conexión de los módulos, es del tipo guirnalda (un cable UTP directo se conecta al puerto de entrada de un módulo y otro cable al puerto de salida del módulo, cada salida del módulo anterior se conecta al puerto de entrada del módulo siguiente; de esta forma se acoplan los módulos hasta que se llegue al último módulo, que es el que actúa como terminador de red). Del módulo que actúa como maestro de la red (módulo PLCpantalla táctil), la conexión va al módulo de expansión de entradas-salidas, y este se une al módulo de arranque directo (que posee un elemento terminador de red). La conexión entre módulos se hace con cable UTP directo (Automatización BP, 2012).

Cada elemento de una red CANopen se identifica mediante un número que se denomina nodo, para el caso de una red en CANopen hay un número máximo de 127 nodos. Entre más pequeña sea la magnitud del número de nodo, el elemento que le corresponde a dicho nodo tiene mayor prioridad en la comunicación. Por ejemplo, en la red implementada, el número uno (la magnitud más pequeña) corresponde al maestro de la red (PLC); entre más grande sea la prioridad del elemento de red, este elemento será el primero al que se le actualicen los estados de sus entradas y salidas, y así sucesivamente, dependiendo de la prioridad de los demás elementos de la red.

CANopen soporta los modelos de comunicación punto a punto, maestro esclavo y productor-consumidor, en sus variantes push y pull. En el modelo push, los productores colocan los eventos en el canal de eventos y este se los envía a los consumidores. En el pull, el flujo de eventos ocurre en el sentido contrario, es decir, los consumidores solicitan eventos al canal de eventos y este los pide a los productores (Sánchez, 2007). Para la red implementada, el modelo de comunicación usado corresponde a uno maestro esclavo, el maestro (PLC) envía y recibe datos a los esclavos de la red (pantalla táctil, módulo de expansión de entradas salidas y módulo de arranque directo).

\section{CONCLUSIONES}

Con este trabajo, no solo se entrega una herramienta de emulación de un proceso a disposición de los usuarios de los equipos del laboratorio de automatización industrial de la UPTC Sogamoso, también se proporcionan guías instructivas, a fin de que los usuarios de los equipos tengan una base bibliográfica para que ellos mismos puedan adelantar aplicaciones de supervisión, control y adquisición de datos con los módulos disponibles. 
Los sistemas SCADA realizados para pantallas táctiles, tienen las mismas caracteristicas funcionales que los sistemas diseñados para computadores industriales, aunque dependen de la gama del panel táctil usado. La diferencia entre los dos sistemas radica en que los primeros son usados para controles locales aplicados a máquinas o procesos puntuales, mientras que los segundos son usados para controles remotos que engloban gran número de sistemas de control local (unión de procesos).

Los sistemas de supervisión, control y adquisición de datos en pantallas táctiles, han reemplazado actualmente muchos sistemas de accionamiento eléctrico y electromecánico de procesos, representando un elemento práctico, de uso espacial reducido y con una gama extensa de herramientas.

La función ejecutada por los autores, tiene la ventaja que puede ser usada con los elementos conectados (módulos, sensores) o sin los elementos conectados, esto le entrega al usuario un proceso que puede utilizar tanto para simulación (elementos no conectados) como para emulación (elementos conectados).

Es fundamental tener en cuenta la asignación correcta del número de nodo en cada elemento usado en una red CANopen, ya que este es el identificador de dispositivo en la red implementada, así como la configuración de los parámetros de comunicación en el dispositivo maestro de la red, destinando los archivos identificadores de elemento de red, que para el caso de una red CANopen corresponden a los archivos EDS (Electronic Data Sheet).

\section{REFERENCIAS}

\section{Adamo, F., Attivissimo, F., Cavone, G. \&} Giaquinto, N. (2005). SCADA/HMI Systems in Advanced Educational Courses. IMTC 2005, Instrumentation and Measurement Technology Conference. Ottawa, Canada.

Automatización BP. (2012). Guías de laboratorio Módulo didáctico de PLC EC4P. Sogamoso, Colombia: UPTC Seccional Sogamo.

Bejan, C., Jacob, M. \& Andreescu, G.-D. (2009).

SCADA Automation System Laboratory, Elements and Applications. (2009). IEEE Intelligent Systems and Informatics. SISY 2009. 7th International Symposium.

Bohórquez, M. \& Salamanca, C. (2012). Guías 1, 2,

3,4, 5 para desarrollo de sistemas de supervisión control y adquisición de datos aplicados a la emulación de procesos en pantalla táctil industrial. Recuperado de http://www.uptc.edu.co/facultades/f_sogamoso/ pregrado/electronica/inf_adicional/aspectos_ac ademicos/index.html.

Goodwin, G., Medioli, A., Sher, W., Ljubo B., Vlacic, L. \& Welsh, J.S. (2011). Emulation-Based Virtual Laboratories: A Low-Cost Alternative to Physical Experiments in Control Engineering Education. Callaghan, NSW 2308, Callaghan, NSW 2308, Australia: The University of Newcastle.

Montalvo, J. \& Morocho, W. (2011). Diseño e implementación de un sistema SCADA para control del proceso de un módulo didáctico de montaje FESTO utilizando PLCy una pantalla HMI, caso práctico: En el laboratorio de automatización de la FIE. Tesis de pregrado. Escuela

Superior Politécnica de Chimborazo. Facultad de Informática y Electrónica. Escuela de Ingeniería Electrónica en Control y

Redes Industriales, p. 18-23.

Parr, E.A. (2003). Programmable Controllers an Engineer's Guide. Oxford, England: Elsevier, p. 101.

Rodríguez, A. (2007). Sistemas SCADA. Notas de diseño, normativa, seguridady comunicaciones industriales, Primeros pasos con InTouch. Barcelona, España: Marcombo, p.p. 21-23.

Sánchez, R. (2007). CANopen. Salamanca, España: Universidad de Salamanca.

User Manual Galileo 4.2 Software. (2012). (Capítulos 2, 3, 6, 7, 8, 9, 10, 15, 16). Micro Innovation AG.

User Manual for PLC Programming with CoDeSys 2.3. (2012). Smart Software Solutions GmbH. (Capítulos $2,5,7)$
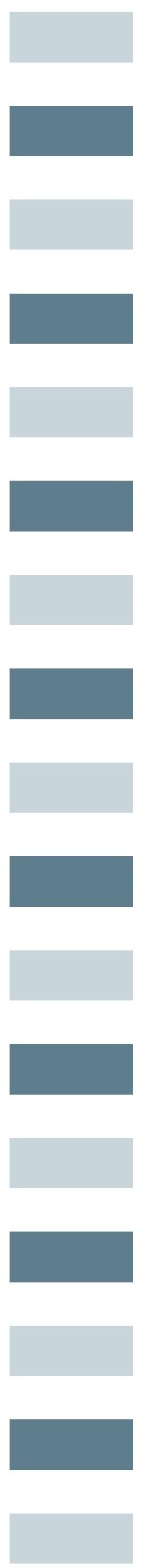